\title{
Internet of Things (IoT): Interconnected Network of Sensors Measuring Physiological Parameters
}

\author{
Anil Umesh ${ }^{1}$ \\ BE, Department of CSE, SJCE, Mysore, India ${ }^{1}$
}

\begin{abstract}
Internet of things (IOT) is a network or an ecosystem of interconnected items embedded with electronics, software and sensors connected with the internet based on stipulated protocols. One of the defining feature of IoT in the present day is that they are no longer exclusively tied to major providers. Smaller networks can exist if they are practical. IoT creates these small networks between its system devices. Devices have become smaller, cheaper and robust overtime. Medical sensors measuring various physiological parameters of the body holds greater advantage in exploiting the concept and working of IoT. In this paper we employ body temperature sensor, pulse sensor, ECG and GPS to track the location of patient. The values of the above sensors are uploaded to the cloud via Wi-Fi module. A graph of these values is plotted and analysed using IoT forum called 'thingspeak.com' providing dedicated channels to each user logging in with a unique user-ID and Password. Data can be any time exported into CSV, XML and JSON format.
\end{abstract}

Keywords: Internet of things (IOT), body temperature sensor, pulse sensor, ECG, GPS, CSV, XML and JSON format.

\section{INTRODUCTION}

An ecosystem of interconnected medical devices measuring physiological parameters like human body temperature, pulse rate, ECG of a person, saturation level and many more critical parameters and running on stipulated protocols constitute IoT. In this paper we have built a network of following sensors/electronics at the nodes measuring

- Pulse rate

- Human body temperature

- $\quad$ ECG pattern

Today, IoT can dramatically enhance medical research and emergency care. The integration of all elements provide more accuracy, more attention to detail, faster reactions to events, and constant improvement while reducing the typical overhead of medical research and organizations.

\section{ENABLING NETWORK TECHNOLOGIES}

The choice of a network technology depends mainly on the span of the network. In this project a Body Area Network (BAN) is employed and is highly secure and restricted within particular bands of frequency and bandwidth. Wi-Fi device is used to transmit the data to the cloud. Data collected from multiple devices come in different formats and at different sampling rates. ETL (Extraction, Transformation, Loading) tools aggregate, process and store data in a format that can be used for analytics application.

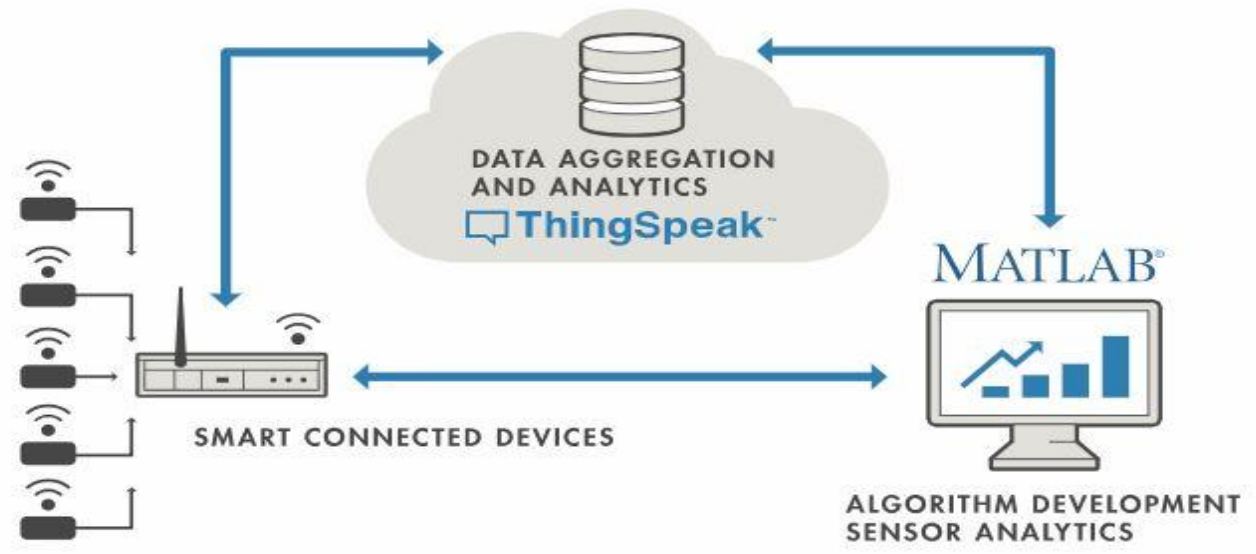

Figure 3 shows the block diagram of IoT using Thingspeak for data aggregation and analytics. 


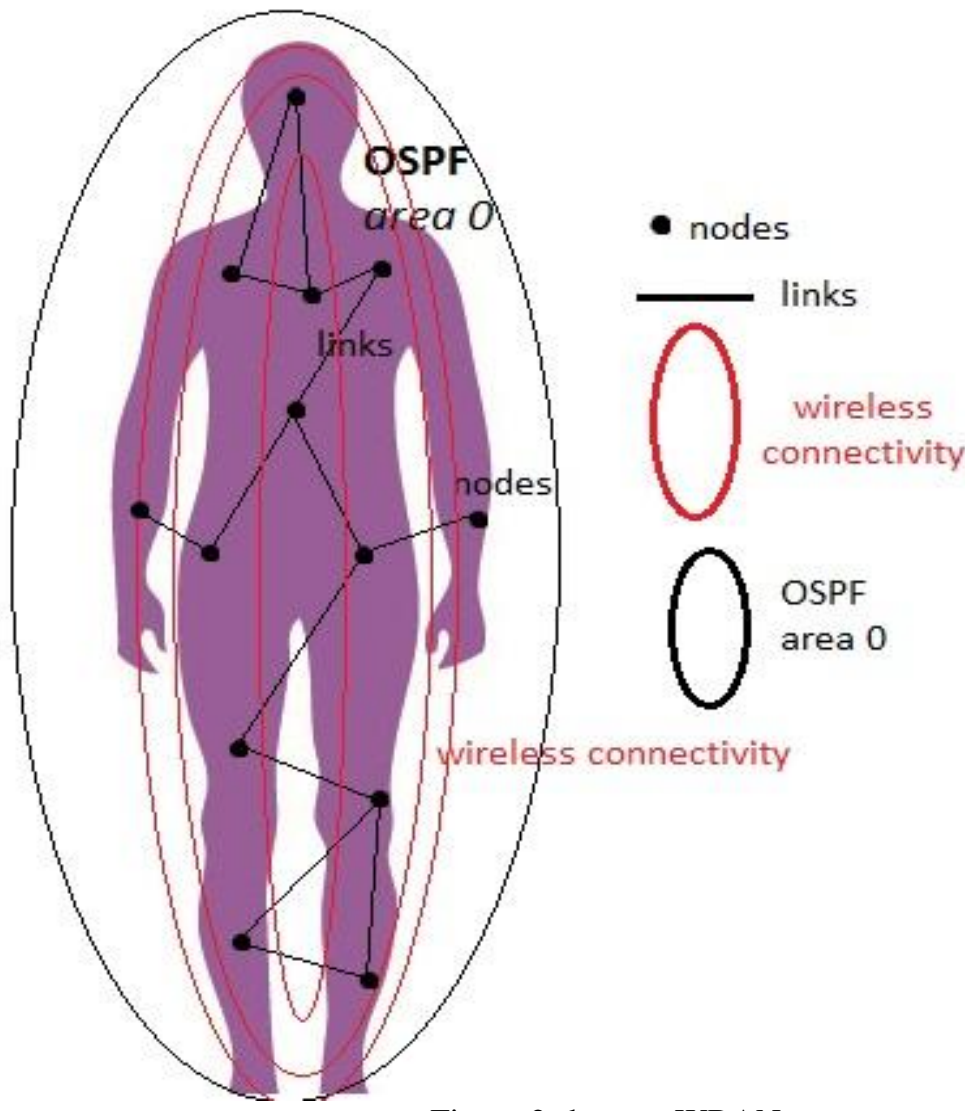

Figure 2 shows a WBAN

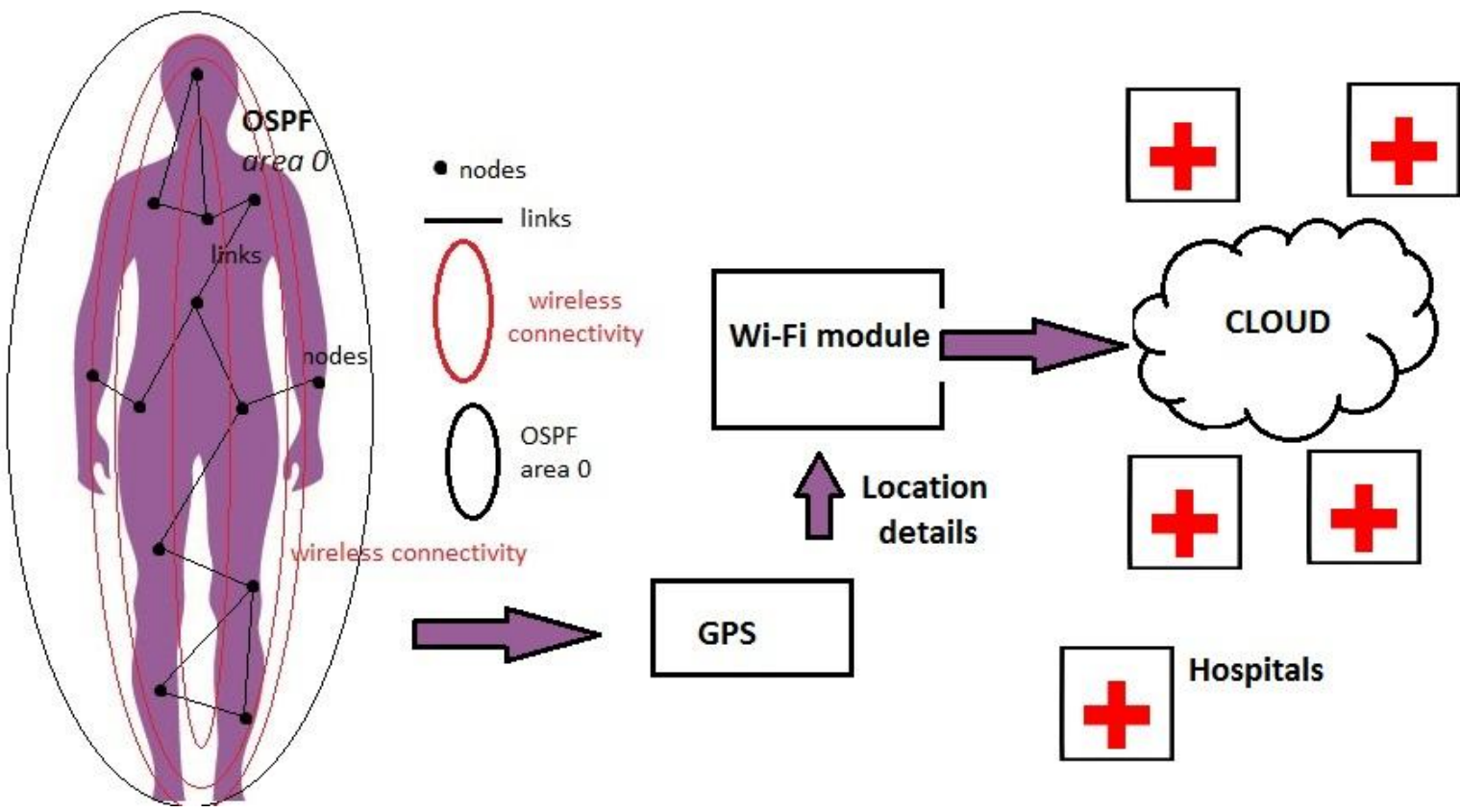

Figure 1 shows the system design of IoT and the data being circulated within the system.

\section{SYSTEM DESIGN}

System design describes the system to be built to operate (do a specific job) and achieve a specific goal. Figure 1 shows the system design of IoT and the data being circulated within the system. It consists of a wireless sensor network or Wireless Body Area Network running wireless protocols as shown in figure 2. Few of the sensors placed at the nodes are pulse sensor, ECG and human body temperature sensor. The data from sensors is uploaded to the cloud via Wi-Fi module. A GPS unit is used to track the live location of the patient in case of emergency. Physiological parameters of the patient can be accessed from the cloud by doctors/hospitals. Overall, an interconnected network of electronics, 
software and sensors is established and can any day serve as a source of analytical data. Figure 3 shows the block diagram of IoT using Thingspeak for data aggregation and analytics. "Thingspeak.com" provides a dedicated channel for data acquisition and analysis. Any registered user can login with his/her unique email-ID and password. Few of the features of Thingspeak are [1]

- Collect data in private channels

- Share data with public channels

- MATLAB analytics and visualisation

- Worldwide community

Thingspeak works with

- Arduino [2]

- $\quad$ ESP8266 Wi-Fi module [3]

- Raspberry Pi [4]

- Mobile and web apps

\section{Results}

Various experiments were conducted and suitable results are published. Figure 4, 5, and 6 shows the human body temperature, pulse rate and ECG of a subject at different instants. Figure 7 shows the location of the subject. Figure 8 shows the ECG discrete values.

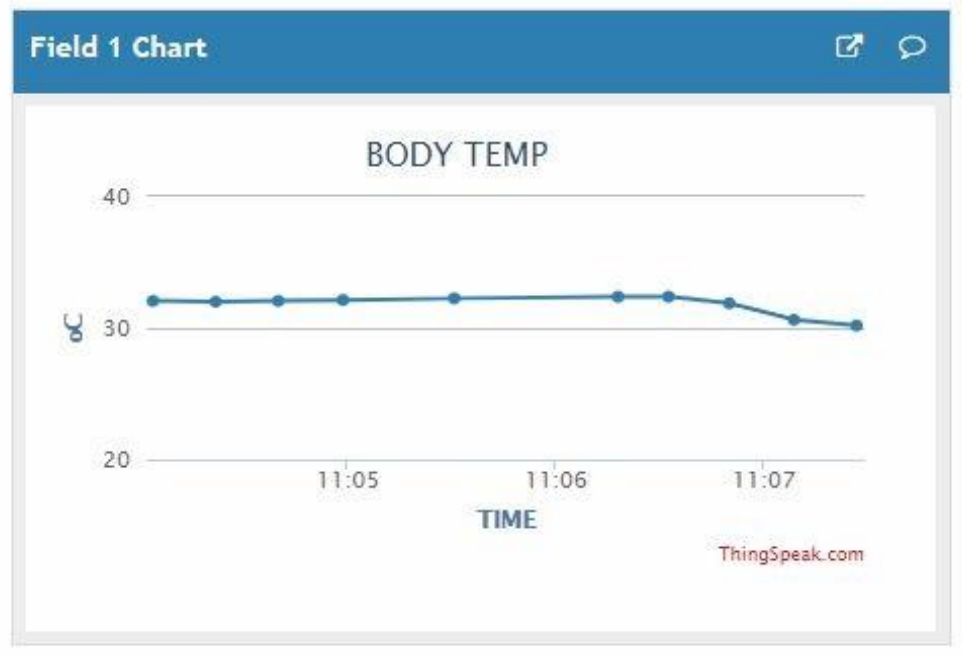

Figure 4 shows human body temperature of the subject

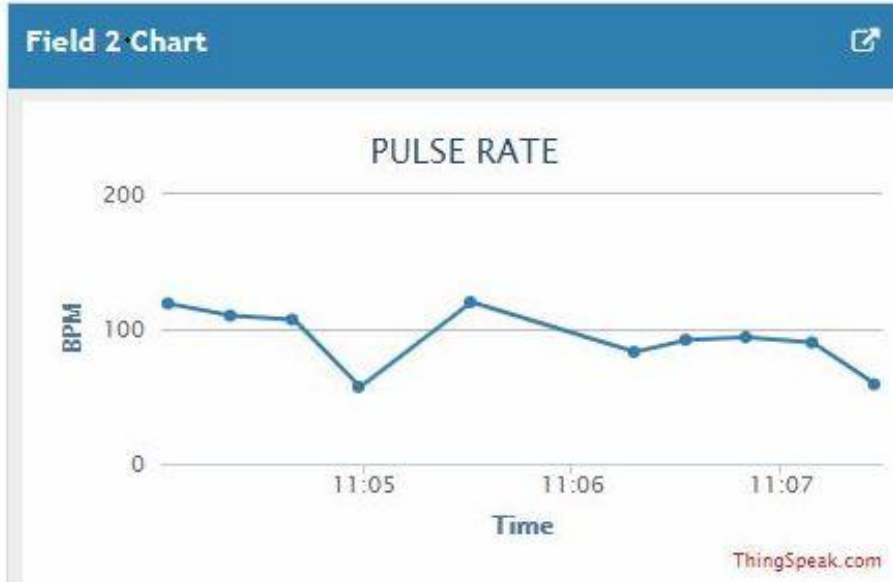

Figure 5 shows pulse rate of the subject 


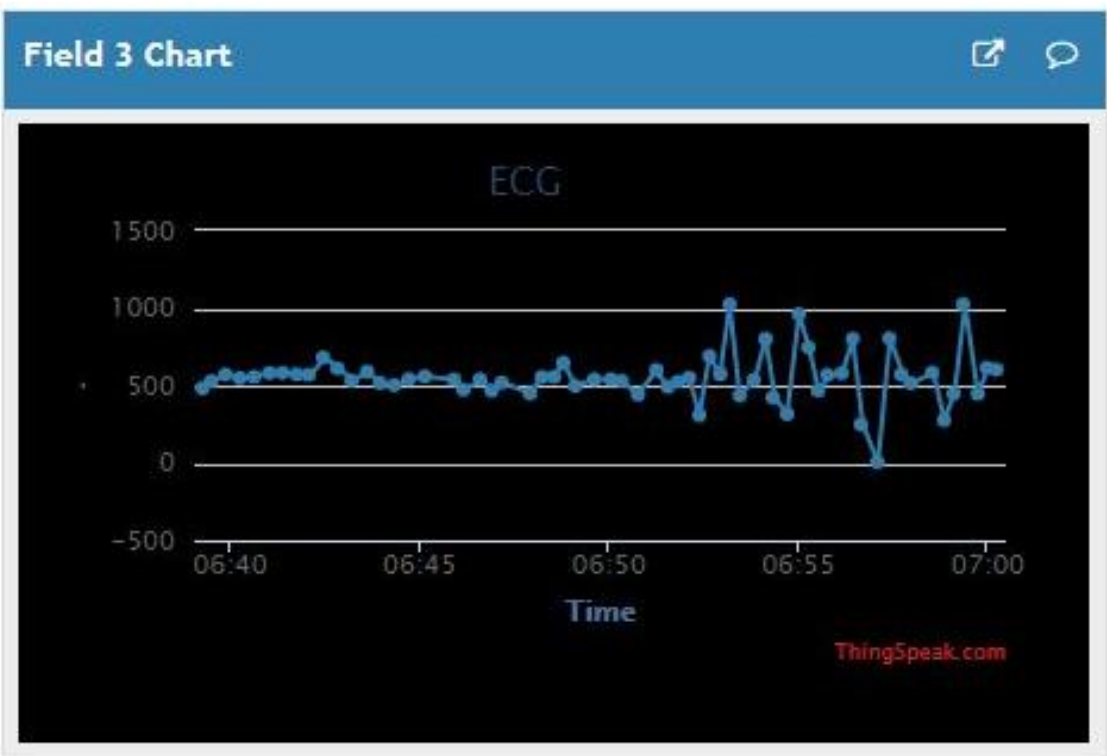

Figure 6 shows the ECG pattern of the subject

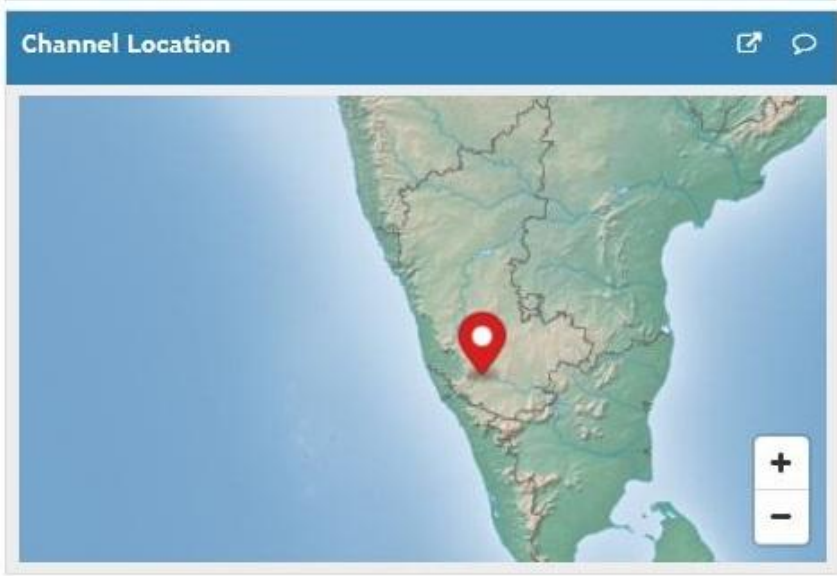

Figure 7 shows the location of the patient

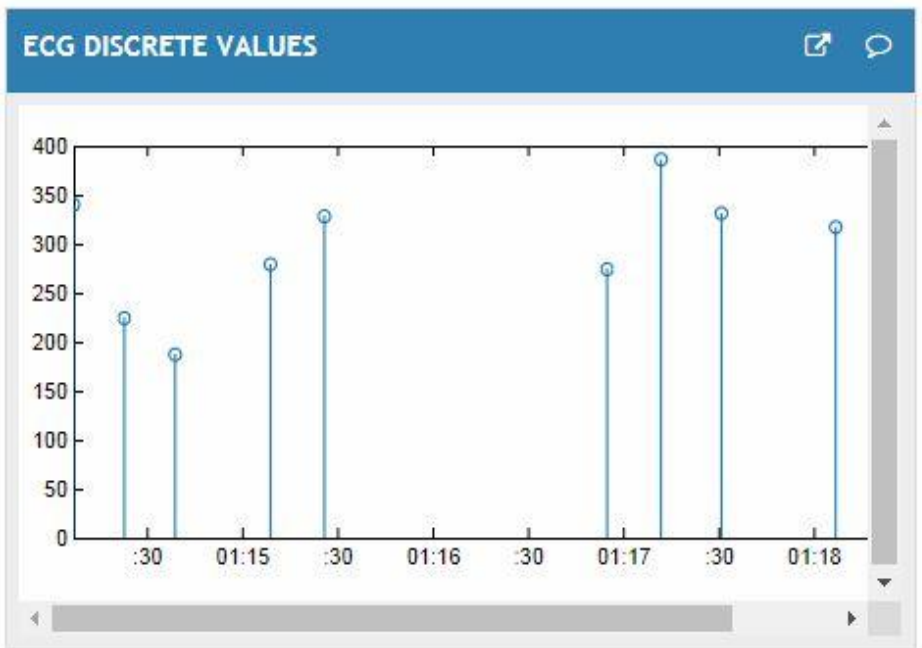

Figure 8 shows the ECG discrete values 


\section{CONCLUSIONS}

In this paper efforts were put in to build an interconnected network of electronics, software and sensors measuring vital human physiological parameters such as pulse rate, human body temperature and ECG. The data from these sensors were uploaded to the cloud using a Wi-Fi module and the live location of the subject was predicted using a GPS module. The hospitals or health care centres have access to these critical data and suitable action can be taken during emergency. The data can any time be exported into one of the mentioned formats- CSV, JSON or XML.

\section{REFERENCES}

[1] ThingSpeak ${ }^{\mathrm{TM}}$ - https://thingspeak.com/

[2] Arduino- https://www.arduino.cc/

[3] ESP8266 Datasheet - ElectroSchematics.com- https://www.electroschematics.com/11276/esp8266-datasheet/

[4] Raspberry Pi — Teach, Learn, and Make with Raspberry Pi- https://www.raspberrypi.org

\section{OUR GUIDE}

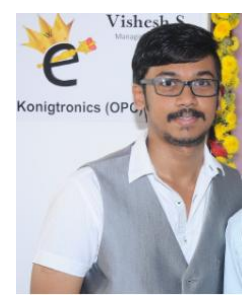

VISHESH S born on $13^{\text {th }}$ June 1992, hails from Bangalore (Karnataka) and has completed B.E in Telecommunication Engineering from VTU, Belgaum, Karnataka in 2015. He also worked as an intern under Dr. Shivananju BN, former Research Scholar, Department of Instrumentation, IISc, Bangalore. His research interests include Embedded Systems, Wireless Communication, BAN and Medical Electronics. He is also the Founder and Managing Director of the corporate company Konigtronics Private Limited. He has guided over a hundred students/interns/professionals in their research work and projects. He is also the co-author of many International Research Papers. He is currently pursuing his MBA in e-Business and PG Diploma in International Business. Presently Konigtronics Private Limited has extended its services in the field of Software Engineering and Webpage Designing. Konigtronics also conducts technical and non-technical workshops on various topics. 